\title{
Paths in Graphs and Curves on Surfaces
}

\author{
Alexander Schrijver
}

\section{Introduction}

A classical result in graph theory due to the topologist Menger [24] concerns the existence of pairwise internally vertex-disjoint paths connecting two given vertices $r$ and $s$ in an undirected graph $G=(V, E)$. (Two paths are internally vertex-disjoint if they do not have any vertex or edge in common, except for the end vertices.)

Menger's theorem states that the maximum number of such paths is equal to the minimum size of a set $W$ of vertices with $r, s \notin W$ such that each path from $r$ to $s$ intersects $W$ at least once. (It is assumed that $r$ and $s$ are not adjacent.) The interest of Menger in this problem originated from characterizing the bifurcation number of certain topological spaces which he named Kurven.

A similar theorem holds if we replace 'undirected' by 'directed'. Moreover, variants are obtained by replacing 'internally vertex-disjoint' by 'edgedisjoint'. (Two paths are edge-disjoint if they do not have any edge in common.)

Menger's theorem is a basic result in graph theory, and several other theorems in graph theory utilize Menger's theorem in some way. Application in optimization followed when Ford and Fulkerson [6] proved their famous max-flow min-cut theorem: the maximum amount of 'flow' that can be transmitted from some 'source' $r$ to some 'sink' $s$ in a capacitated network, is equal to the minimum capacity of any $r-s$ cut. (An $r-s$ cut is a set of edges intersecting each path from $r$ to s.) This theorem can be derived from Menger's theorem (and vice versa).

Ford and Fulkerson [7] also designed a fast algorithm to determine a maximum flow from $r$ to $s$. This method formed the basis for a wealth of applications in operations research, e.g., to problems involving assignment, transportation, transshipment, routing, circulation, and communication.

Menger's theorem and the max-flow min-cut theorem are of interest also because they provide us with a way of 'certifying' that a certain path packing or a certain flow is the largest possible. Indeed, to convince somebody that a certain collection of $k$ pairwise internally vertex-disjoint $r-s$ paths is the largest possible, it suffices to exhibit a set $W$ of vertices intersecting each $r-s$ path and satisfying $|W|=k$ - by Menger's theorem that such a set always exists. In the language of complexity theory, this 
implies that the problem of finding a maximum path packing belongs to the complexity class NPกco-NP. In other words, Menger's theorem is a 'good characterization.'

Similarly, the optimality of a certain $r-s$ flow can be shown by displaying an $r-s$ cut with capacity equal to the value of the flow. Again it implies that the the problem of finding a maximum flow belongs to NPกco-NP. So paths and flows connecting one given pair $r, s$ of vertices in a network are 'well under control.'

The situation changes however if we need disjoint paths or flows each of which connects a different pair of vertices. This is often the case in practice, when one is not interested in connecting only one pair of source and sink in a network by paths or by a flow, but several pairs of sources and sinks simultaneously.

One may think of a large communication or transportation network, where several messages or goods must be transmitted all at the same time over the network between different pairs of terminals. A recent application is the design of very large-scale integrated (VLSI) circuits, where several pairs of pins must be interconnected by wires on a chip in such a way that the wires follow given 'channels' and the wires connecting different pairs of pins do not intersect each other.

Mathematically, this gives the disjoint paths problem:

given: a graph $G=(V, E)$ and vertices $r_{1}, s_{1}, \ldots, r_{k}, s_{k}$ of $G$;

find: $\quad$ pairwise disjoint paths $P_{1}, \ldots, P_{k}$, where $P_{i}$ runs from $r_{i}$ to $s_{i}(i=1, \ldots, k)$.

Here a graph can be undirected or directed. Pairwise disjoint can mean: pairwise vertex-disjoint (i.e., any two paths do not have any vertex in common) or pairwise edge-disjoint. So there are four variants of Problem (1).

It turns out that these problems are much less tractable than when we would have only one pair of source and sink. In fact, D.E. Knuth (cf. [14]) and Lynch [23] proved that each of the variants is NP-complete, even when we restrict ourselves to planar graphs. If $\mathrm{NP} \neq \mathrm{co}-\mathrm{NP}$, this fact wrecks the hopes for a polynomial-time algorithm solving the disjoint paths problem and for a good characterization of the existence of disjoint paths, e.g. in the spirit of Menger's theorem.

On the other hand, it is a deep result of Robertson and Seymour [31] that, for each fixed $k$, the undirected variants of the disjoint paths problem can be solved in polynomial time, in fact in time $O\left(|V|^{3}\right)$. The constant in this bound depends heavily on $k$, so the result does not imply $\mathrm{P}=\mathrm{NP}$. (Recently, B.A. Reed improved the complexity to $O\left(|V|^{2} \log |V|\right)$.)

The result of Robertson and Seymour is one of the products of the 
great Graph Minors project, with as a landmark the theorem that each infinite collection of undirected graphs contains two graphs $G, H$ so that $G$ is a 'minor' of $H$ (that is, $G$ can be obtained from $H$ by deletion and contraction of edges).

Part of the proof method of Robertson and Seymour is based on embedding a graph on a surface. This makes paths and minors in the graph easier to handle by using the topology of the surface, for instance, by classifying paths (and more generally graphs) on the surface by their homotopic properties.

In fact, also in the study of the disjoint paths problem for designing chips, the idea of utilizing the topology of the chip surface came up. There one considers the 'modules' placed on the chip as holes in the surface, and one searches for a layout of disjoint paths (wires) as required by enumerating (or gucssing) homotopy types of such paths. We refer to Pinter [27], Cole and Siegel [2], and Leiserson and Maley [20].

The directed versions of the disjoint paths problem are NP-complete even when we fix $k$ equal to 2 , as was shown by Fortune, Hopcroft, and Wyllie [8]. So this is in contrast (for those believing $\mathrm{P} \neq \mathrm{NP}$ ) to Robertson and Seymour's theorem for the undirected case.

In [45] it is shown that, for any fixed $k$, the vertex-disjoint paths problem is solvable in polynomial time for directed planar graphs. More generally, it suffices to fix the number of faces that are necessary to cover the terminals (by their boundaries), and to restrict the directed graphs to those embeddable on some fixed compact surface. We do not know the complexity of the edge-disjoint paths problem for directed planar graphs if we fix $k$ equal to 2 .

The results of Robertson and Seymour and the applications to the design of chips have enhanced the research in disjoint paths problems and their relations to curves and graphs embedded on surfaces. In this paper we give an overview of some of the results. We focus on graphs embedded on compact surfaces and derive results on disjoint paths with a strong bias on our own results. (In particular, we do not survey the fundamental results of $\mathrm{Hu}$ [13], Karzanov [15], [16], Lomonosov [22], Seymour [48] and others on edgc-disjoint paths and multicommodity flows in general graphs with few terminals.)

In this paper, a path may traverse a vertex or edge more than once. However, by convention, 'pairwise vertex-disjoint' is meant to imply that each of the paths traverses any vertex (and hence any edge) at most once; similarly, 'pairwise edge-disjoint' is meant to imply that each of the paths traverses any edge at most once. If a graph is directed we call its edges arcs. 


\section{The cohomology feasibility problem}

We first consider vertex-disjoint paths in graphs on surfaces. The following framework turned out to be helpful.

Let $D=(V, A)$ be a directed graph and let $G$ be a group. Two functions $\phi, \psi: A \longrightarrow G$ are called cohomologous if there exists a function $p: V \longrightarrow$ $G$ such that

$$
\psi(a)=p(u)^{-1} \phi(a) p(w)
$$

for each $\operatorname{arc} a=(u, w)$ of $D$. One directly checks that this is an equivalence relation.

Consider the following cohomology feasibility problem:

given: a directed graph $D=(V, A)$, a group $G$, and functions $\phi: A \longrightarrow G$ and $H: A \longrightarrow \mathcal{P}(G)$;

find: a function $\psi: A \longrightarrow G$ such that $\psi$ is cohomologous to $\phi$ and such that $\psi(a) \in H(a)$ for each $a \in A$.

$[\mathcal{P}(G)$ is the collection of all subsets of $G$.]

There is an obvious necessary condition for the existence of such a function $\psi$. Let us denote a path $P$ in $D$ as a word $a_{1} \cdots a_{t}$ over the alphabet $\left\{a, a^{-1} \mid a \in A\right\}$. In this way we indicate that $P$ traverses the arcs $a_{1}, \ldots, a_{t}$ in this order, where $a_{i}=a^{-1}$ means that arc $a$ is traversed in backward direction. A $v-w$ path is a path starting in $v$ and ending in $w$.

Define $\phi\left(a^{-1}\right):=\phi(a)^{-1}$ and $H\left(a^{-1}\right):=H(a)^{-1}$. For any path $P=$ $a_{1} \cdots a_{t}$ define $\phi(P):=\phi\left(a_{1}\right) \cdots \phi\left(a_{t}\right) \in G$ and $H(P):=H\left(a_{1}\right) \cdots H\left(a_{t}\right) \subseteq$ G.

A necessary condition for the existence of $\psi$ in the cohomology feasibility problem (3) is:

for each $v \in V$ and each $v-v$ path $P$ there exists an $x \in G$ such that $x^{-1} \phi(P) x \in H(P)$.

Indeed, we can take $x=p(v)$ where $p$ satisfies (2).

In some cases this condition is sufficient as well, for instance, if $G$ is the infinite group with one generator $g$ and each $H(a)$ is convex (that is, if $g^{i}, g^{j} \in H(a)$ then also $g^{k} \in H(a)$ whenever $k$ is inbetween $i$ and $j$ ).

However, this condition generally is not sufficient (see the Remark below). A stronger necessary condition is:

for each $v \in V$ and each two $v-v$ paths $P_{1}, P_{2}$ there exists an $x \in G$ such that $x^{-1} \phi\left(P_{1}\right) x \in H\left(P_{1}\right)$ and $x^{-1} \phi\left(P_{2}\right) x \in H\left(P_{2}\right)$, 
since again we can take $x=p(v)$.

We are going to give a class of groups, the graph groups (or free partially commutative groups), for which, for certain subsets $H(a)$, condition (5) is sufficient. (Graph groups are studied, inter alia, in [1], [5], and [46], but we do not make use of these results.)

A graph group is constructed as follows. Let $g_{1}, \ldots, g_{k}$ be generators, and let $E$ be a collection of pairs $\{i, j\}$ with $i, j \in\{1, \ldots, k\}$ and $i \neq$ $j$. Then the group $G=G_{k, E}$ is the group generated by $g_{1}, \ldots, g_{k}$ with relations

$$
g_{i} g_{j}=g_{j} g_{i} \text { for each }\{i, j\} \in E .
$$

So if $E=\emptyset$ then $G_{k, E}$ is the free group generated by $g_{1}, \ldots, g_{k}$, while if $E$ consists of all pairs from $\{1, \ldots, k\}$ then $G_{k, E}$ is isomorphic to $\mathbb{Z}^{k}$.

There is the following direct reduction rule for words over the 'symbols' $g_{1}, g_{1}^{-1}, \ldots, g_{k}, g_{k}^{-1}$ : if symbol $\alpha$ commutes with each symbol occurring in word $y$, then $x \alpha y \alpha^{-1} z=x y z$. It can be shown that repeating this reduction as long as possible starting with a word $w$, one reaches the empty word 1 if $w$ equals 1 in the group. So the word problem can be solved easily (cf. [50]).

Applying this reduction to a general word $w$, one obtains a shortest possible word $w^{\prime}$ (shortest among all words $w^{\prime \prime}$ that are equal to $w$ in the group). The length of $w^{\prime}$ is denoted by $|w|$. This defines a 'norm' on $G_{k, E}$, satisfying $|1|=0,\left|u^{-1}\right|=|u|$ and $|u w| \leq|u|+|w|$. So we can define a distance function dist on $G$ by:

$$
\operatorname{dist}(x, y):=\left|x^{-1} y\right|
$$

for $x, y \in G$. For $x, y \in G$ let $[x, y]$ be the set of all $z \in G$ satisfying $\operatorname{dist}(x, z)+\operatorname{dist}(z, y)=\operatorname{dist}(x, y)$.

Call a subset $H$ of $G$ closed if

(i) $1 \in H$,

(ii) $[x, y] \subseteq H$ for all $x, y \in H$,

(iii) $[x, y] \subseteq H^{-1}$ for all $x, y \in H^{-1}$

Note that if $G$ is the free group then $H \subseteq G$ is closed if and only if $H \neq \emptyset$ and for each reduced word $w \in H$, also each subsegment of $w$ belongs to $H$.

In [45] the following theorem is proved. 
Theorem 1. Let $G$ be a graph group and let each $H(a)$ be closed. Then the cohomology feasibility problem (3) has a solution $\psi$ if and only if condition (5) is satisfied.

The proof is based on a polynomial-time algorithm giving either the function $\psi$ or a pair of paths $P_{1}, P_{2}$ violating (5). Therefore we also have:

Theorem 2. The cohomology feasibility problem (3) is solvable in polynomial time if $G$ is a graph group and each $H(a)$ is closed.

We assume here that membership of $H(a)$ of a given word can be checked in polynomial time.

Remark. We cannot relax condition (5) in Theorem 1 to condition (4). To see this, let $G$ be the free group generated by $g$ and $h$. Let $D$ be the directed graph with one vertex $v$ and two loops, $a$ and $b$, attached at $v$. Define $\phi(a):=h, H(a):=\left\{1, h, g, g^{-1}, g^{-1} h, h g\right\}$ and $\phi(b):=g h g^{-1}, H(b):=$ $\left\{1, h, g, g^{-1}, h g^{-1}, g h\right\}$. If $x^{-1} \phi(a) x \in H(a)$ then the first symbol of $x$ is not equal to $g$. If $x^{-1} \phi(b) x^{-1} \in H(b)$ then the first symbol of $x$ is equal to $g$. So there is no $x$ such that both hold.

On the other hand, for each path $P$ in $D$ there is an $x$ such that $x^{-1} \phi(P) x \in H(P)$. Indeed, for each $k \in \mathbb{Z}, \phi\left(a b^{k}\right) \in H\left(a b^{k}\right)$ and $\phi\left(b^{k} a\right) \in$ $H\left(b^{k} a\right)$. It follows that if $P$ starts or ends with $a$ or $a^{-1}$, then $\phi(P) \in H(P)$. Moreover, for each $k \in \mathbb{Z}, g^{-1} \phi\left(a^{k} b\right) g \in H\left(a^{k} b\right)$ and $g^{-1} \phi\left(b a^{k}\right) g \in H\left(b a^{k}\right)$. So if $P$ starts and ends with $b$ or $b^{-1}$ then $g^{-1} \phi(P) g \in H(P)$.

\section{The $k$-disjoint paths problem for directed planar graphs}

We first sketch how the polynomial-time solvability of the cohomology feasibility problem for closed subsets of graph groups implies that, for each fixed $k$, the $k$ disjoint paths problem for directed planar graphs:

given: a directed planar graph $D=(V, A)$ and $k$ pairs $\left(r_{1}, s_{1}\right), \ldots,\left(r_{k}, s_{k}\right)$ of vertices of $D$;

find: $k$ pairwise vertex-disjoint directed paths $P_{1}, \ldots, P_{k}$ in $D$, where $P_{i}$ runs from $r_{i}$ to $s_{i}(i=1, \ldots, k)$.

is solvable in polynomial time $([45])$.

Theorem 3. For each fixed $k$, the $k$ disjoint paths problem for directed planar graphs (9) is solvable in polynomial time.

We sketch the proof. As graph group $G$ we take the free group with $k$ 
generators $g_{1}, \ldots, g_{k}$.

Let input $D=(V, A), r_{1}, s_{1}, \ldots, r_{k}, s_{k} \in V$ for (9) be given. We may assume that $D$ is weakly connected, and that $r_{1}, s_{1}, \ldots, r_{k}, s_{k}$ are distinct, each being incident with exactly one arc. Fix an embedding of $D$, and let $\mathcal{F}$ denote the collection of faces of $D$.

Call two functions $\phi, \psi: A \longrightarrow G$ homologous if there exists a function $f: \mathcal{F} \longrightarrow \mathcal{G}$ such that

$$
f(F)^{-1} \phi(a) f\left(F^{\prime}\right)=\psi(a)
$$

for each $\operatorname{arc} a$, where $F$ and $F^{\prime}$ are the faces at the left-hand side and at the right-hand side of $a$, respectively.

For any solution $\Pi=\left(P_{1}, \ldots, P_{k}\right)$ of $(9)$ let $\phi_{\Pi}: A \longrightarrow G$ be defined by:

$$
\begin{aligned}
\phi_{\Pi}(a):=g_{i} & \text { if path } P_{i} \text { traverses } a(i=1, \ldots, k), \text { and } \\
\phi_{\Pi}(a):=1 & \text { if } a \text { is not traversed by any of the } P_{i}
\end{aligned}
$$

Now one can show:

For each fixed $k$, we can find in polynomial time functions $\phi_{1}, \ldots, \phi_{N}: A \longrightarrow G$ with the property that for each solution II of $(9), \phi_{\mathrm{II}}$ is homologous to at least one of $\phi_{1}, \ldots, \phi_{N}$.

[This is the only reason why we can prove the polynomial-time solvability of Problem (9) for fixed $k$ only.]

It follows that it suffices to describe a polynomial-time method for the following problem:

given: a function $\phi: A \longrightarrow G$;

find: a solution $\Pi$ of (9) such that $\phi_{\Pi}$ is homologous to $\phi$.

Indeed, we can apply such an algorithm to each $\phi_{j}$ in (12). If we find no $\Pi$ for any $\phi_{j},(9)$ has no solution.

In order to solve (13) with the cohomology feasibility algorithm, we consider the dual graph $D^{*}=\left(\mathcal{F}, \mathcal{A}^{*}\right)$ of $D$, having as vertex set the collection $\mathcal{F}$ of faces of $D$, while for any $\operatorname{arc} a$ of $D$ there is an $\operatorname{arc}$ of $D^{*}$, denoted by $a^{*}$, from the face of $D$ at the left-hand side of $a$ to the face at the right-hand side of $a$. (So the dual graph may have loops.)

Define for any function $\phi$ on $A$ the function $\phi^{*}$ on $A^{*}$ by

$$
\phi^{*}\left(a^{*}\right):=\phi(a)
$$


for each $a \in A$. Then any two functions $\phi$ and $\psi$ are homologous (in $D$ ) if and only if $\phi^{*}$ and $\psi^{*}$ are cohomologous (in $D^{*}$ ).

We extend the dual graph to the 'extended' dual graph $D^{+}=\left(\mathcal{F}, \mathcal{A}^{+}\right)$ by adding in each face of $D^{*}$ all chords. (So $D^{+}$need not be planar.) To be more precise, for any two vertices $F, F^{\prime}$ of $D^{*}$ and any (undirected) $F-F^{\prime}$ path $\pi$ on the boundary of any face of $D^{*}$, extend $D^{*}$ with an arc, called $a_{\pi}$, from $F$ to $F^{\prime}$. For any $\phi: A \longrightarrow G$ define $\phi^{+}: A^{+} \longrightarrow G$ by:

$$
\begin{aligned}
& \phi^{+}\left(a^{*}\right):=\phi^{*}\left(a^{*}\right) \text { for each arc } a \text { of } D \\
& \phi^{+}\left(a_{\pi}\right):=\phi^{*}(\pi) \text { for any path } \pi \text { as above. }
\end{aligned}
$$

Moreover, let

$$
\begin{aligned}
& H\left(a^{*}\right):=\left\{1, g_{1}, \ldots, g_{k}\right\} \text { and } \\
& H\left(a_{\pi}\right):=\left\{1, g_{1}, g_{1}^{-1}, \ldots, g_{k}, g_{k}^{-1}\right\}
\end{aligned}
$$

So each of these sets is a closed subset of the free group $G$.

Now let input $\phi$ of Problem (13) be given. As the cohomology feasibility problem is solvable in polynomial time in this case (Theorem 2), we can find in polynomial time a function $\psi$ that is cohomologous to $\phi^{+}$in $D^{+}$, with $\psi(b) \in H(b)$ for each $\operatorname{arc} b$ of $D^{+}$, provided that such a $\psi$ exists. If we find one, let $P_{i}$ be any directed $r_{i}-s_{i}$ path traversing only arcs a satisfying $\psi\left(a^{*}\right)=g_{i}(i=1, \ldots, k)$. If such paths exist, they form a solution to the disjoint paths problem (9).

If we do not find such a function $\psi$ and such paths we may conclude that Problem (13) has no solution. For suppose that $\phi_{\Pi}$ is homotopic to $\phi$ for some $\Pi:=\left(P_{1}, \ldots, P_{k}\right)$. Then there exists a $\psi$ as above, viz. $\psi:=\left(\phi_{\Pi}\right)^{+}$. Moreover, for any $\psi^{\prime}$ cohomologous to $\left(\phi_{\Pi}\right)^{+}$there exists for each $i=1, \ldots, k$ a directed $r_{i}-s_{i}$ path $P_{i}^{\prime}$ traversing only arcs $a$ such that $g_{i}$ occurs in $\psi^{\prime}\left(a^{*}\right)$. So we would find a solution, contradicting our assumption.

This finishes the sketch of the proof of Theorem 3 .

\section{Further applications to disjoint paths}

First, by extending (12) we obtain the following generalization. For any directed graph $G=(V, A)$ embedded on a compact surface $S$, and any subset $X$ of $V$, let $\tau(X)$ denote the minimum number $t$ for which there exist faces $F_{1}, \ldots, F_{t}$ of $D$ such that $X \subseteq \operatorname{bd}\left(F_{1} \cup \cdots \cup F_{t}\right)$. Then we have:

Theorem 4. For each fixed t, the disjoint paths problem for directed 
planar graphs (9) is solvable in polynomial time for inputs satisfying $\tau\left(\left\{r_{1}, s_{1}, \ldots, r_{k}, s_{k}\right\}\right) \leq t$.

This can be shown as (12) can be extended to fixing

$$
\tau\left(\left\{r_{1}, s_{1}, \ldots, r_{k}, s_{k}\right\}\right) \leq t,
$$

instead of fixing $k$. Theorem 4 extends a theorem of Robertson and Seymour [30] for undirected planar graphs with $t=2$. (Recently, Ripphausen, Wagner, and Weihe [29] gave a linear-time algorithm for undirected planar graphs with $t=2$.)

One may also derive that the following problem:

given: a directed planar graph $D=(V, A), k$ pairs $\left(r_{1}, s_{1}\right), \ldots$, $\left(r_{k}, s_{k}\right)$ of vertices of $D$, and subsets $A_{1}, \ldots, A_{k}$ of $A$;

find: $\quad k$ pairwise vertex-disjoint directed paths $P_{1}, \ldots, P_{k}$ in $D$, where $P_{i}$ runs from $r_{i}$ to $s_{i}$ and uses only arcs in $A_{i}$ $(i=1, \ldots, k)$,

is solvable in polynomial time, for fixed $k$. This follows by restricting in (16) the $H\left(a^{*}\right)$ to those $g_{i}$ for which $A_{i}$ contains $a$.

Theorem 5. For each fixed $t$, problem (17) is solvable in polynomial time for inputs satisfying $\tau\left(\left\{r_{1}, s_{1}, \ldots, r_{k}, s_{k}\right\}\right) \leq t$.

More generally, consider the problem:

given: a directed planar graph $D=(V, A), k$ pairs $\left(r_{1}, s_{1}\right)$ $\ldots,\left(r_{k}, s_{k}\right)$ of vertices of $D$, subsets $A_{1}, \ldots, A_{k}$ of $A$, and a set $E$ consisting of some pairs $\{i, j\}$ from $1, \ldots, k$;

find: $\quad k$ directed paths $P_{1}, \ldots, P_{k}$ in $D$, where $P_{i}$ runs from $r_{i}$ to $s_{i}$ and uses only $\operatorname{arcs}$ in $A_{i}(i=1, \ldots, k)$ and where $P_{i}$ and $P_{j}$ are disjoint if $\{i, j\} \notin E$.

Theorem 6. For each fixed $k$, problem (18) is solvable in polynomial time.

Moreover, one has:

Theorem 7. Problem (18) is solvable in polynomial time for inputs satisfying $\tau\left(\left\{r_{1}, s_{1}, \ldots, r_{k}, s_{k}\right\}\right) \leq 2$.

This follows as above from the polynomial-time solvability of the cohomology feasibility problem applied to the graph group $G_{k, E}$. It is unknown to me if Theorem 7 holds for any fized bound $t$ on $\tau\left(\left\{r_{1}, s_{1}, \ldots, r_{k}, s_{k}\right\}\right)$. 
This result was shown for $t=1$ by Ding, Schrijver, and Seymour [3].

Similar is the following disjoint trees problem:

given: a directed planar graph $D=(V, A)$ and $k$ pairs $\left(r_{1}, S_{1}\right)$, $\ldots,\left(r_{k}, S_{k}\right)$ with $r_{1}, \ldots, r_{k} \in V$ and $S_{1}, \ldots, S_{k} \subseteq V$

find: $k$ pairwise vertex-disjoint directed rooted trees $T_{1}, \ldots, T_{k}$ in $D$, where $T_{i}$ has root $r_{i}$ and covers $S_{i}(i=1, \ldots, k)$.

Here we say that a tree covers a set $S$ if $S$ is a subset of the vertex set of $T$.

Corollary 6a. For each fixed t, problem (19) is solvable in polynomial time for inputs satisfying $\tau\left(\left\{r_{1}, \ldots, r_{k}\right\} \cup S_{1} \cup \cdots \cup S_{k}\right) \leq t$.

This follows from Theorem (6) by taking all pairs $\left(r_{i}, s^{\prime}\right)$ with $i \in$ $\{1, \ldots, k\}$ and $s^{\prime} \in S_{i}$, and defining $E$ so that directed paths will be disjoint if they correspond to distinct $r_{i}$ and $r_{j}$.

These polynomial-time solvability results for directed planar graphs, in fact also hold for any directed graph embedded on some compact surface $S$, as long as we keep this surface fixed. We mention the following:

given: a directed graph $D=(V, A)$ embedded on a compact surface and $k$ pairs $\left(r_{1}, s_{1}\right), \ldots,\left(r_{k}, s_{k}\right)$ of vertices of $D$;

find: $k$ pairwise vertex-disjoint directed paths $P_{1}, \ldots, P_{k}$ in $D$, where $P_{i}$ runs from $r_{i}$ to $s_{i}(i=1, \ldots, k)$.

Theorem 8. For each fixed $k$ and each fixed compact surface $S$, the $k$ disjoint paths problem for directed graphs embedded on $S$ (20) is solvable in polynomial time.

One of the most general results in this direction concerns the following problem:

given: a directed graph $D=(V, A)$ embedded on a compact surface $S, k$ pairs $\left(r_{1}, s_{1}\right), \ldots,\left(r_{k}, s_{k}\right)$ of vertices of $D$, subsets $A_{1}, \ldots, A_{k}$ of $A$, and a set $E$ of pairs $\{i, j\}$ from $\{1, \ldots, k\}$

find: $\quad k$ directed paths $P_{1}, \ldots, P_{k}$ in $D$, where $P_{i}$ runs from $r_{i}$ to $s_{i}$ and uses only $\operatorname{arcs}$ in $A_{i}(i=1, \ldots, k)$, and where $P_{i}$ and $P_{j}$ are vertex-disjoint if $\{i, j\} \notin E$.

Theorem 9. For each fixed compact surface $S$ and each fixed $k$, problem (21) is solvable in polynomial time. 
The proof method can also be applied if we fix homotopies. For instance, the following disjoint homotopic paths problem is solvable in polynomial time, not fixing $k$ or $p$ :

given: a directed planar graph $G$ embedded in the plane $\mathbb{R}^{2}$, faces $F_{1}, \ldots, F_{p}$ of $G$ (including the unbounded face), and curves $C_{1}, \ldots, C_{k}$ in $S:=\mathbb{R}^{2} \backslash\left(F_{1} \cup \cdots \cup F_{p}\right)$;

find: pairwise vertex-disjoint simple directed paths $P_{1}, \ldots, P_{k}$ in $G$ so that for each $i=1, \ldots, k, P_{i}$ is homotopic to $C_{i}$ in $S$.

As usual, a curve is a continuous function $C:[0,1] \longrightarrow S$. Two curves $C, C^{\prime}$ are homotopic, in notation $C \sim C^{\prime}$, if there exists a continuous function $\Phi:[0,1] \times[0,1] \longrightarrow S$ such that $\Phi(x, 0)=C(x), \Phi(x, 1)=C^{\prime}(x), \Phi(0, x)=$ $C(0)$ and $\Phi(1, x)=C(1)$ for each $x \in[0,1]$.

This result extends a theorem for finite subgraphs of the rectangular grid in the plane of Leiserson and Maley [20] and one for undirected planar graphs of Schrijver [39].

\section{Disjoint closed curves in graphs on a compact surface}

Let $S$ be a surface. A closed curve on $S$ is a continuous function $C: S^{1} \longrightarrow$ $S$, where $S^{1}$ is the unit circle in $\mathbb{C}$. Two closed curves $C$ and $C^{\prime}$ are called freely homotopic, in notation $C \sim C^{\prime}$, if there exists a continuous function $\Phi: S^{1} \times[0,1] \longrightarrow S$ such that $\Phi(z, 0)=C(z)$ and $\Phi(z, 1)=C^{\prime}(z)$ for each $z \in S^{1}$.

For any pair of closed curves $C, D$ on $S$, let $\operatorname{cr}(C, D)$ denote the number of crossings of $C$ and $D$, counting multiplicities. Moreover, $\operatorname{mincr}(C, D)$ denotes the minimum of $\operatorname{cr}\left(C^{\prime}, D^{\prime}\right)$ where $C^{\prime}$ and $D^{\prime}$ range over closed curves freely homotopic to $C$ and $D$, respectively. That is,

$$
\operatorname{mincr}(C, D):=\min \left\{\operatorname{kr}\left(C^{\prime}, D^{\prime}\right) \mid C^{\prime} \sim C, D^{\prime} \sim D\right\} .
$$

Let $G=(V, E)$ be an undirected graph embedded on $S$. (We identify $G$ with its embedding on $S$.) For any closed curve $D$ on $S, \operatorname{cr}(G, D)$ denotes the number of intersections of $G$ and $D$ (counting multiplicities):

$$
\operatorname{cr}(G, D):=\left|\left\{z \in S^{1} \mid D(z) \in G\right\}\right| .
$$

The following was shown in [38] (motivated by [31]):

Theorem 10. Let $G=(V, E)$ be an undirected graph embedded on a 
compact surface $S$ and let $C_{1}, \ldots, C_{k}$ be pairwise disjoint simple closed curves on $S$, each nonnullhomotopic. Then there exist pairwise vertexdisjoint simple circuits $C_{1}^{\prime}, \ldots, C_{k}^{\prime}$ in $G$ such that $C_{i}^{\prime} \sim C_{i}(i=1, \ldots, k)$, if and only if for each closed curve $D$ on $S$ :

$$
\operatorname{cr}(G, D) \geq \sum_{i=1}^{k} \operatorname{mincr}\left(C_{i}, D\right)
$$

with strict inequality if $D$ is doubly odd.

Here we call a closed curve $D$ on $S$ doubly odd (with respect to $G$ and $C_{1}, \ldots, C_{k}$ ) if $D$ is the concatenation $D_{1} \cdot D_{2}$ of two closed curves $D_{1}$ and $D_{2}$ such that $D_{1}(1)=D_{2}(1) \notin G$ and such that

$$
\operatorname{cr}\left(G, D_{j}\right) \not \equiv \sum_{i=1}^{k} \operatorname{cr}\left(C_{i}, D_{j}\right) \quad(\bmod 2)
$$

for $j=1,2$.

It is easy to see that the condition in the theorem is necessary, since if $C_{1}^{\prime}, \ldots, C_{k}^{\prime}$ exist and $D$ is a closed curve on $S$ then

$$
\operatorname{cr}(G, D) \geq \sum_{i=1}^{k} \operatorname{cr}\left(C_{i}^{\prime}, D\right) \geq \sum_{i=1}^{k} \operatorname{mincr}\left(C_{i}, D\right)
$$

If $D$ is doubly odd, then taking $D_{1}$ and $D_{2}$ as above we have for cach $i=1, \ldots, k$ :

$$
\operatorname{cr}\left(C_{i}^{\prime}, D_{1}\right) \equiv \operatorname{cr}\left(C_{i}, D_{1}\right) \quad(\bmod 2)
$$

since the parity of the number of crossings of two curves is invariant under homotopic transformations. Hence by (26),

$$
\operatorname{cr}\left(G, D_{1}\right) \not \equiv \sum_{i=1}^{k} \operatorname{cr}\left(C_{i}^{\prime}, D_{1}\right) \quad(\bmod 2) \text {. }
$$

Since $C_{1}^{\prime}, \ldots, C_{k}^{\prime}$ are pairwise disjoint we know

$$
\operatorname{cr}\left(G, D_{1}\right) \geq \sum_{i=1}^{k} \operatorname{cr}\left(C_{i}^{\prime}, D_{1}\right)
$$

and hence by (29) we should have strict inequality here. Therefore

$$
\operatorname{cr}(G, D)=\operatorname{cr}\left(G, D_{1}\right)+\operatorname{cr}\left(G, D_{2}\right)>\sum_{i=1}^{k} \operatorname{cr}\left(C_{i}^{\prime}, D_{1}\right)+\sum_{i=1}^{k} \operatorname{cr}\left(C_{i}^{\prime}, D_{2}\right)
$$




$$
=\sum_{i=1}^{k} \operatorname{cr}\left(C_{i}^{\prime}, D_{1} \cdot D_{2}\right)=\sum_{i=1}^{k} \operatorname{cr}\left(C_{i}^{\prime}, D\right) \geq \sum_{i=1}^{k} \operatorname{mincr}\left(C_{i}, D\right) .
$$

The essence of the theorem is sufficiency of the condition.

The theorem can be extended to directed circuits in directed graphs embedded on a compact orientable surface, although the condition becomes more difficult to describe. (For the torus, see Seymour [48], cf. [4].) In any case, the method yields a polynomial-time algorithm finding the directed circuits.

\section{Decomposition of graphs on surfaces}

We now shift our attention from vertex-disjointness to edge-disjointness. Edge-disjoint paths and curves on a surface are more difficult to keep under control as they can cross each other as often as they like.

De Graaf and Schrijver [11] showed the following result, which was proved for the projective plane by Lins [21] (see Corollary 16a below) and for compact orientable surfaces by Schrijver [37]. The theorem implies a fractional packing result of closed curves to be discussed in the next section.

For any graph $G=(V, E)$ embedded on a surface $S$ and any closed curve $D$ let $\operatorname{mincr}(G, D)$ denote the minimum value of $\operatorname{cr}\left(G, D^{\prime}\right)$ where $D^{\prime}$ ranges over all closed curves $D^{\prime} \sim D$ not intersecting $V$.

Theorem 11. Let $G=(V, E)$ be an Eulerian graph embedded on a surface $S$. Then the edges of $G$ can be decomposed into closed curves $C_{1}, \ldots, C_{k}$ such that

$$
\operatorname{mincr}(G, D)=\sum_{i=1}^{k} \operatorname{mincr}\left(C_{i}, D\right)
$$

for each closed curve $D$ on $S$.

Here a graph is Eulerian if each vertex has even degree. (We do not assume connectedness of the graph.) Moreover, decomposing the edges into $C_{1}, \ldots, C_{k}$ means that for each $s \in G \backslash V$ there is exactly one pair $(i, z)$ such that $C_{i}(z)=s$; for $s \notin G$ there is no such pair at all, while for $s \in V$ there are $\frac{1}{2} \operatorname{deg}(s)$ such pairs. $(\operatorname{deg}(s)$ denotes the degree of $s$.) We assume the surface is triangulizable.

The inequality $\geq$ in (32) trivially holds, for any decomposition of the edges into closed curves $C_{1}, \ldots, C_{k}$ : by definition of $\operatorname{mincr}(G, D)$, there exists a closed curve $D^{\prime} \sim D$ in $S \backslash V$ such that $\operatorname{mincr}(G, D)=\operatorname{cr}\left(G, D^{\prime}\right)$, and hence

$$
\operatorname{mincr}(G, D)=\operatorname{cr}\left(G, D^{\prime}\right)=\sum_{i=1}^{k} \operatorname{cr}\left(C_{i}, D^{\prime}\right) \geq \sum_{i=1}^{k} \operatorname{mincr}\left(C_{i}, D\right) .
$$


The content of the theorem is that there exists a decomposition attaining equality. For a proof we refer to [11].

Using surface duality one can derive the following from Theorem 11 . If $G$ is a graph embedded on a surface $S$ and $C$ is a closed curve in $G$, then minlength $_{G}(C)$ denotes the minimum length of any closed curve $C^{\prime} \sim C$ in $G$. (The length of $C^{\prime}$ is the number of edges traversed by $C^{\prime}$, counting multiplicities.)

Corollary 11a. Let $G=(V, E)$ be a bipartite graph embedded on a compact surface $S$ and let $C_{1}, \ldots, C_{k}$ be closed curves in $G$. Then there exist closed curves $D_{1}, \ldots, D_{t}$ on $S \backslash V$ such that each edge of $G$ is crossed by exactly one $D_{j}$ and by this $D_{j}$ only once and such that

$$
\operatorname{minlength}_{G}\left(C_{i}\right)=\sum_{j=1}^{t} \operatorname{mincr}\left(C_{i}, D_{j}\right)
$$

for each $i=1, \ldots, k$.

In fact we may replace the bipartiteness condition by the condition that all faces are even.

\section{Homotopic circulations}

We derive a fractional packing result from Theorem 11. Let $G=(V, E)$ be an undirected graph embedded on a compact surface $S$, and let $C_{1}, \ldots, C_{k}$ be closed curves on $S$. We are interested in the existence of pairwise edgedisjoint closed curves $C_{1}^{\prime}, \ldots, C_{k}^{\prime}$ in $G$ such that $C_{i}^{\prime} \sim C_{i}$ for each $i=$ $1, \ldots, k$. (Here 'pairwise edge-disjoint' includes that each $C_{i}^{\prime}$ should traverse any edge at most once.) A necessary condition for the existence of such curves is:

cut condition: for each closed curve $D$ on $S \backslash V$ :

$$
\text { cr }(G, D) \geq \sum_{i=1}^{k} \operatorname{mincr}\left(C_{i}, D\right) \text {. }
$$

This condition generally is not sufficient. However, it is sufficient for the existence of a 'fractional packing' of such closed curves.

Call a function (or vector) $f \in \mathbb{R}_{+}^{E}$ a circulation (of value 1 ) if $f$ is a convex combination of functions $\operatorname{tr}_{C^{\prime}}$, where $C^{\prime}$ is a closed curve in $G$, and where $\operatorname{tr}_{C^{\prime}}$ denotes the function in $\mathbb{R}_{+}^{E}$ given by: $\operatorname{tr}_{C^{\prime}}(e):=$ the number of times $C^{\prime}$ traverses edge $e$.

We say that $f$ is freely homotopic to a closed curve $C$ if we can take each $C^{\prime}$ freely homotopic to $C$. 
Note that if $f$ is a circulation freely homotopic to $C$, then for each closed curve $D$ on $S \backslash V$ one has (denoting by $\operatorname{cr}(e, D)$ the number of times $D$ intersects edge $e$ ):

$$
\sum_{e \in E} f(e) \operatorname{cr}(e, D) \geq \operatorname{mincr}(C, D) .
$$

This follows from the fact that (36) holds for $f:=\operatorname{tr}_{C^{\prime}}$ for each $C^{\prime}$ freely homotopic to $C$ (as $\sum_{e \in E} \operatorname{tr}_{C^{\prime}}(e) \operatorname{cr}(e, D)=\operatorname{cr}\left(C^{\prime}, D\right) \geq \operatorname{mincr}(C, D)$ ), and hence also for each convex combination of such vectors.

We now derive the result of [11]:

Corollary 11b. (Homotopic circulation theorem.) Let $G=(V, E)$ be an undirected graph embedded on a compact surface $S$ and let $C_{1}, \ldots, C_{k}$ be closed curves on $S$. Then there exist circulations $f_{1}, \ldots, f_{k}$ such that $f_{i}$ is freely homotopic to $C_{i}(i=1, \ldots, k)$ and such that $\sum_{i=1}^{k} f_{i}(e) \leq 1$ for each edge $e$, if and only if the cut condition (35) holds.

Note that if each $f_{i}$ would be 0,1 valued, we would have an edge-disjoint collection of closed curves in $G$ of prescribed homotopies.

Proof. Necessity. Suppose there exist circulations $f_{1}, \ldots, f_{k}$ as required, and let $D$ be a closed curve on $S \backslash V$. Then by (36):

$$
\begin{gathered}
\operatorname{cr}(G, D)=\sum_{e \in E} \operatorname{cr}(e, D) \geq \sum_{e \in E} \operatorname{cr}(e, D) \sum_{i=1}^{k} f_{i}(e) \\
=\sum_{i=1}^{k} \sum_{e \in E} f_{i}(e) \operatorname{cr}(e, D) \geq \sum_{i=1}^{k} \operatorname{mincr}\left(C_{i}, D\right) .
\end{gathered}
$$

So the cut condition holds.

Sufficiency. Suppose the cut condition (35) holds. Let $I:=\{1, \ldots, k\}$, and let $K$ be the convex cone in $\mathbb{R}^{I} \times \mathbb{R}^{E}$ generated by the vectors

$$
\begin{gathered}
\left(\varepsilon_{i} ; \operatorname{tr}_{C}\right)\left(i \in I ; C \text { closed curve in } G \text { with } C \sim C_{i}\right) ; \\
\left(0_{I} ; \varepsilon_{e}\right)(e \in E) .
\end{gathered}
$$

Here $\varepsilon_{i}$ denotes the $i$ th unit basis vector in $\mathbb{R}^{I}$ and $\varepsilon_{e}$ denotes the eth unit basis vector in $\mathbb{R}^{E}$. Moreover, $0_{I}$ denotes the all-zero vector in $\mathbb{R}^{I}$.

We must show that the vector $\left(1_{I} ; 1_{E}\right)$ belongs to $K$. Here $1_{I}$ and $1_{E}$ denote the all-one vectors in $\mathbb{R}^{I}$ and $\mathbb{R}^{E}$, respectively. By Farkas' lemma (cf. [33]), it suffices to show that each vector $(d ; l) \in \mathbb{Q}^{I} \times \mathbb{Q}^{E}$ having nonnegative inner product with each of the vectors (38), also has nonnegative 
inner product with $\left(1_{I} ; 1_{E}\right)$. (Farkas' lemma applies, since $K$ is finitely generated, as one easily proves.)

Thus let $(d ; l) \in \mathbb{Q}^{I} \times \mathbb{Q}^{E}$ have nonnegative inner product with each vector among (38). This is equivalent to:

(i) $d_{i}+\sum_{e \in E} l(e) \operatorname{tr}_{C}(e) \geq 0(i \in I ; C$ closed curve in $G$ with $\left.C \sim C_{i}\right)$

(ii) $l(e) \geq 0(e \in E)$.

Suppose that $(d ; l)^{T}\left(1_{I} ; 1_{E}\right)<0$. By increasing $l$ slightly, we may assume that $l(e)>0$ for each $e \in E$. Next, by blowing up $(d ; l)$ we may assume that each entry in $(d ; l)$ is an even integer.

Let $G^{\prime}$ be the graph arising from $G$ by replacing each edge $e$ of $G$ by a path of length $l(e)$. That is, we insert $l(e)-1$ new vertices on $e$. Then by (39(i)),

$$
-d_{i} \leq \operatorname{minlength}_{G^{\prime}}\left(C_{i}\right)
$$

for each $i \in I$. Since $G^{\prime}$ is bipartite, by Corollary 11a there exist closed curves $D_{1}, \ldots, D_{t}$ not intersecting any vertex of $G^{\prime}$ such that each edge of $G^{\prime}$ is intersected by exactly one $D_{j}$ and only once by that $D_{j}$ and such that

$$
\operatorname{minlength}_{G^{\prime}}\left(C_{i}\right)=\sum_{j=1}^{t} \operatorname{mincr}\left(C_{i}, D_{j}\right)
$$

for each $i \in I$. So

$$
l(e)=\sum_{j=1}^{t} \operatorname{cr}\left(e, D_{j}\right)
$$

for each edge $e$ of $G$. Hence (35), (40) and (41) give

$$
\begin{aligned}
& \sum_{e \in E} l(e)=\sum_{j=1}^{t} \sum_{e \in E} \operatorname{cr}\left(e, D_{j}\right)=\sum_{j=1}^{t} \operatorname{cr}\left(G, D_{j}\right) \geq \sum_{j=1}^{t} \sum_{i=1}^{k} \operatorname{mincr}\left(C_{i}, D_{j}\right) \\
& =\sum_{i=1}^{k} \sum_{j=1}^{t} \operatorname{mincr}\left(C_{i}, D_{j}\right)=\sum_{i=1}^{k} \operatorname{minlength}_{G^{\prime}}\left(C_{i}\right) \geq-\sum_{i=1}^{k} d_{i} .
\end{aligned}
$$

So $(d ; l)^{T}\left(1_{I} ; 1_{E}\right) \geq 0$.

In [37] an example is given showing that generally we cannot take the $f_{i} 0,1$ valued, even not if certain parity conditions are satisfied. However, 
Frank and Schrijver [9] showed that if $S$ is the torus, $C_{1}, \ldots, C_{k}$ are simple closed curves, and for each closed curve $D$ one has the 'parity condition'

$$
\operatorname{mincr}(G, D) \equiv \sum_{i=1}^{k} \operatorname{mincr}\left(C_{i}, D\right) \quad(\bmod 2)
$$

then the $f_{i}$ can be taken to be 0,1 valued. That is, there is the following theorem:

Theorem 12. Let $G=(V, E)$ be a graph embedded on the torus $S$, and let $C_{1}, \ldots, C_{k}$ be simple closed curves on $S$, such that condition (44) holds. Then there exist pairwise edge-disjoint closed curves $C_{1}^{\prime}, \ldots, C_{k}^{\prime}$ in $G$ such that $C_{i}^{\prime} \sim C_{i}$ for $i=1, \ldots, k$, if and only if the cut condition (35) holds.

For more results on curves and graphs on the torus, and their relations to geometry of numbers, see [10], [43], [44], [42], and [49].

\section{Homotopic flows}

As a further application we derive a 'homotopic flow-cut theorem' for planar graphs. Let $G=(V, E)$ be an undirected planar graph embedded in the plane $\mathbb{R}^{2}$, let $F_{1}, \ldots, F_{p}$ be some of its faces, including the unbounded face. Define $S:=\mathbb{R}^{2} \backslash\left(F_{1} \cup \cdots \cup F_{p}\right)$. A curve is a continuous function from a closed interval into $S$. So curves have end points, while closed curves have not. Throughout, we take homotopy of curves in the space $S$.

We define the function mincr for curves with end points similarly as for closed curves. Thus, for any pair of curves $C, D$, define $\operatorname{mincr}(C, D)$ to be the minimum number of intersections of $C^{\prime}$ and $D^{\prime}$ (counting multiplicities), where $C^{\prime}$ and $D^{\prime}$ range over all curves on $S$ homotopic to $C$ and $D$, respectively. Moreover, $\operatorname{mincr}(G, D)$ denotes the minimum number of intersections of $G$ and $D^{\prime}$ (counting multiplicities) where $D^{\prime}$ ranges over all curves on $S$ homotopic to $D$ not intersecting $V$.

Let $C_{1}, \ldots, C_{k}$ be curves with end points on bd $(S)$. A direct necessary condition for the existence of pairwise edge-disjoint paths $P_{1}, \ldots, P_{k}$ in $G$ such that $P_{i} \sim C_{i}$ for $i=1, \ldots, k$ is:

cut condition: for each curve $D$ on $S \backslash V$ with end points on $\operatorname{bd}(S)$ :

$$
\operatorname{cr}(G, D) \geq \sum_{i=1}^{k} \operatorname{mincr}\left(C_{i}, D\right)
$$

This condition generally is not sufficient for the existence of paths 
$P_{1}, \ldots, P_{k}$, but it is sufficient for a 'fractional packing' of such paths. For this we need the concept of a flow.

Let $r$ and $s$ be vertices of $G$. Call a function $f: E \longrightarrow \mathbb{R}_{+}$an $r-s$ flow (of value 1) if $f$ is a convex combination of vectors $\operatorname{tr}_{P}$, where $P$ is an $r-s$ path in $G$, and where $\operatorname{tr}_{P}(e)$ denotes the number of times $P$ traverses edge $e$. We say that $f$ is homotopic to some $r-s$ curve $C$ if we can take each $P$ homotopic to $C$ (in $S$ ).

Corollary 11c. Let $C_{1}, \ldots, C_{k}$ be curves in $S$ with end vertices on $\mathrm{bd}(S)$. Then there exist flows $f_{1}, \ldots, f_{k}$ such that $f_{i}$ is homotopic to $C_{i}(i=$ $1, \ldots, k)$ and such that $\sum_{i=1}^{k} f_{i}(e) \leq 1$ for each edge $e$, if and only if the cut condition (45) holds.

This can be derived from Corollary $11 \mathrm{~b}$ by transforming $S$ to a compact surface $S^{\prime}$, by adding a 'handle' $H_{i}$ for each curve $C_{i}$ between the two faces among $F_{1}, \ldots, F_{p}$ having the end points of $C_{i}$ on their boundaries. We then extend $C_{i}$ over $H_{i}$ to a closed curve $C_{i}^{\prime}$ in $S^{\prime}$, and extend $G$ similarly with an edge over $H_{i}$ connecting the end points of $C_{i}$; this gives the graph $G^{\prime}$ embedded on $S^{\prime}$. Applying Corollary $11 \mathrm{~b}$ to $S^{\prime}, G^{\prime}$ and the $C_{i}^{\prime}$ gives Corollary 11c.

One similarly derives a version with 'demands' and 'capacities.' A function $f: E \longrightarrow \mathbb{R}_{+}$is called an $r-s$ flow of value $d$ if $1 / d \cdot f$ is an $r-s$ flow of value 1 . Moreover, such a flow is homotopic to a curve $C$ if $(1 / d) f$ is homotopic to $C$.

Corollary 11d. Let $C_{1}, \ldots, C_{k}$ be curves in $S$ with end vertices on the $\operatorname{bd}(S)$, let $c: E \longrightarrow \mathbb{R}_{+}$(the 'capacity function') and let $d_{1}, \ldots, d_{k} \in \mathbb{R}_{+}$ (the 'demands'). Then there exist flows $f_{1}, \ldots, f_{k}$ such that $f_{i}$ is homotopic to $C_{i}$ and has value $d_{i}(i=1, \ldots, k)$ and such that $\sum_{i=1}^{k} f_{i}(e) \leq c(e)$ for each edge $e$, if and only if for each curve $D$ in $S$ with end points on bd(S) we have

$$
\sum_{i=1}^{k} d_{i} \cdot \operatorname{mincr}\left(C_{i}, D\right) \leq \sum_{e \in E} c(e) \operatorname{cr}(e, D) .
$$

It is not always true that we can take the flows $f_{i}$ to be integer valued. However in some cases this is true, in particular when also the following 'parity condition' is satisfied:

parity condition: for each vertex $v$ of $G$, the degree of $v$ has the same parity as the number of curves $C_{i}$ having $v$ as one of

its end vertices.

The following was shown in [12]: 
Theorem 13. Let the number $p$ of holes be at most 2, and suppose that the parity condition is satisfied. Then there exist pairwise edge-disjoint paths $P_{1}, \ldots, P_{k}$ in $G$ such that $P_{i} \sim C_{i}$ for $i=1, \ldots, k$, if and only if the cut condition (45) holds.

This theorem extends the Okamura-Seymour theorem [26] (where $p=1$ cf. Corollary 11b).

Another case where the cut condition is enough, was shown in [40]:

Theorem 14. Let $G=(V, E)$ be a planar graph and let $F_{1}, \ldots, F_{p}$ be some of its faces, including the unbounded face. Suppose that there exist straight line segments $L_{1}, \ldots, L_{t}$ in the plane such that

$$
G \cup F_{1} \cup \cdots \cup F_{p}=L_{1} \cup \cdots \cup L_{t} \cup F_{1} \cup \cdots \cup F_{p}
$$

and such that each of the $L_{j}$ has its end points in $F_{1} \cup \cdots \cup F_{p}$. Then if the parity condition is satisfied, the cut condition is equivalent to the existence of paths.

This was shown by Kaufmann and Mehlhorn [19] for finite subgraphs of the rectangular grid in the plane.

\section{Primitive closed curves}

Also the following result in combinatorial topology can be derived from Corollary 11 b (cf. [33]). Call a closed curve $D$ on a compact surface $S$ primitive if there does not exist a closed curve $C$ and a natural number $n \geq$ 2 such that $D \sim C^{n}$. Moreover, call two systems of closed curves $C_{1}, \ldots, C_{k}$ and $C_{1}^{\prime}, \ldots, C_{k^{\prime}}^{\prime}$ equivalent if $k=k^{\prime}$ and there exists a permutation $\pi$ of $\{1, \ldots, k\}$ such that $C_{\pi(i)}^{\prime} \sim C_{i}$ or $C_{\pi(i)}^{\prime} \sim C_{i}^{-1}$ for each $i=1, \ldots, k$. Then:

Theorem 15. Two systems of primitive closed curves $C_{1}, \ldots, C_{k}$ and $C_{1}^{\prime}, \ldots, C_{k^{\prime}}^{\prime}$ on a compact surface $S$ are equivalent, if and only if for each closed curve $D$ on $S$

$$
\sum_{i=1}^{k} \operatorname{mincr}\left(C_{i}, D\right)=\sum_{i=1}^{k^{\prime}} \operatorname{mincr}\left(C_{i}^{\prime}, D\right)
$$

The necessity of the condition is trivial - the content of the result is sufficiency. 
It is not difficult to see that one cannot delete the primitivity condition in the theorem. However, one can relax the condition to each curve being 'orientably primitive': a curve $D$ is orientably primitive if there does not exist an orientation-preserving closed curve $C$ and a natural number $n \geq 2$ such that $D \sim C^{n}$. In particular, each orientation-reversing closed curve is orientably primitive. (On orientable surfaces, 'primitive' coincides with 'orientably primitive.')

For an application of this result to the uniqueness of 'kernels' in compact orientable surfaces, see [41].

\section{The Klein bottle}

We finally focus on the Klein bottle and its relations to planar multicommodity flows. Let $G=(V, E)$ be an undirected graph embedded on the Klein bottle $S$. Call a set $B$ of edges of $G$ a blocker if $B$ intersects each orientation-reversing circuit in $G$.

In [36] we proved the following min-max relations:

\section{Theorem 16.}

(i) If $G$ is bipartite, the minimum number of edges in an orientationreversing circuit in $G$ is equal to the maximum number of pairwise disjoint blockers.

(ii) If $G$ is Eulerian, the minimum size of a blocker is equal to the maximum number of pairwise edge-disjoint orientation-reversing circuits.

Again, in (1) we can relax the bipartiteness condition to cach face being even.

As usual, the inequalities $\min \geq \max$ are easy. The content of the theorem is formed by the reverse inequalities. In fact, (ii) can be derived from (i), as we indicate below.

The starting point in the method for proving Theorem 16 is the following result proved in [35]:

Let $G=(V, E)$ be a connected bipartite undirected planar graph, and let $F_{1}$ and $F_{2}$ be two of its faces. Then there exist pairwise disjoint cuts $D_{1}, \ldots, D_{t}$ such that for each two vertices $u, w$ with $u, w \in \operatorname{bd}\left(F_{1}\right)$ or $u, w \in \operatorname{bd}\left(F_{2}\right)$, the distance in $G$ from $u$ to $w$ is equal to the number of cuts $D_{j}$ separating $u$ and $w$.

Here a set $D$ of edges is called a cut if there is a subset $X$ of $V$ such 
that $D=\delta(X):=\{e \in E|| e \cap X \mid=1\}$. The cut $D$ is said to separate $u$ and $w$ if $X$ contains exactly one of $u, w$.

From (50) one derives Theorem 16(i) as follows. First cut the Klein bottle open along a minimum-length orientation-reversing circuit $C$ in $G$. This transforms the Klein bottle $S$ to a Móbius strip $S^{\prime}$, and the graph $G$ to a graph $G^{\prime}$ (in which $C$ is 'doubled').

Next we cut $S^{\prime}$ open along a minimum-length orientation-reversing circuit $C^{\prime}$ in $G^{\prime}$. This transforms the Möbius strip $S^{\prime}$ to an annulus $S^{\prime \prime}$ and the graph $G^{\prime}$ to a graph $G^{\prime \prime}$ (in which $C^{\prime}$ is 'doubled').

As $G^{\prime \prime}$ is a planar bipartite graph, (50) applies to $G^{\prime \prime}$, with the two 'holes' of the annulus taken as the faces $F_{1}$ and $F_{2}$. Then the cuts $D_{1}, \ldots, D_{t}$ can be seen to correspond to a maximum packing of $|C|$ blockers in $G$, and we have Theorem 16(i).

Now by a standard technique in polyhedral combinatorics one derives from Theorem 16(i) that each vertex of the polyhedron in $\mathbb{R}^{E}$ determined by:

(i) $x(e) \geq 0$, for each $e \in E$,

(ii) $\sum_{e \in C} x(e) \geq 1$, for each orientation reversing circuit $C$.

is the incidence vector of some blocker. Linear programming duality applied to minimizing the function $\sum_{e \in E} x(e)$ over (51) then gives the following min-max relation:

the minimum size of a blocker in $G$ is equal to the maximum value of $\sum_{C} y_{C}$, where $y_{C} \in \mathbb{R}_{+}$for each orientation-reversing circuit $C$, such that $\sum_{C \ni e} y_{C} \leq 1$ for each edge $e$ of $G$.

Now Theorem 16(ii) says that if $G$ is Eulerian, then we can take in fact the $y_{C}$ from $\{0,1\}$. This can be derived from (52) by applying a few additional techniques (see [36]). It implies that for general (non-Eulerian) graphs $G$ we can take the $y_{C}$ in (52) from $\left\{0, \frac{1}{2}, 1\right\}$. This follows by replacing each edge by two parallel edges, thus making the graph Eulerian, and then applying Theorem 16(ii).

We mention some consequences of Theorem 16(ii). First the following result of Lins [21] on the projective plane can be derived. (Observe that on the projective plane, the orientation-reversing closed curves are exactly the nonnullhomotopic closed curves.)

Corollary 16a. (Lins' theorem.) Let $G=(V, E)$ be an Eulerian undirected graph embedded on the projective plane. Then the maximum number of pairwise edge-disjoint nonnullhomotopic circuits in $G$ is equal to the 
minimum number of edges intersected by a nonnullhomotopic closed curve not intersecting $V$.

This follows directly by adding an extra cross-cap on the projective plane (in one of the faces of $G$ ), transforming it to a Klein bottle.

Lins' theorem is equivalent to a theorem on disjoint paths in planar graphs, due to Okamura and Seymour [26]. Let $G=(V, E)$ be an undirected graph, and let $r_{1}, s_{1}, \ldots, r_{k}, s_{k}$ be vertices of $G$ (so that $r_{i} \neq s_{i}$ for each $i$ ).

We are interested in the existence of

pairwise edge-disjoint paths $P_{1}, \ldots, P_{k}$ where $P_{i}$ connects

$r_{i}$ and $s_{i}(i=1, \ldots, k)$.

Clearly, the following cut condition is a necessary condition for the existence of such paths:

$$
\text { cut condition: for each } X \subseteq V:|\delta(X)| \geq|\rho(X)| \text {, }
$$

where $\rho(X)$ denotes the set of $i \in\{1, \ldots, k\}$ such that $X$ separates $r_{i}$ and $s_{i}$.

Generally, the cut condition is not sufficient for the existence of the paths. In some cases, the following additional (not-necessary) condition is helpful:

parity condition: $|\delta(\{v\})| \equiv|\rho(\{v\})|(\bmod 2)$, for each vertex $v$.

Corollary 16b. (Okamura-Seymour theorem.) Let $G=(V, E)$ be an undirected planar graph, let $F$ be one of its faces, and let $r_{1}, s_{1}, \ldots, r_{k}, s_{k}$ be vertices on $b d(F)$, so that the parity condition is satisfied. Then the cut condition (54) is equivalent to the existence of paths (53).

In fact also a more general theorem of Okamura [25] can be derived from Theorem 16(ii).

Corollary 16c. (Okamura's theorem.) Let $G=(V, E)$ be an undirected planar graph, let $F_{1}$ and $F_{2}$ be two of its faces, and let $r_{1}, s_{1}, \ldots, r_{k}, s_{k}$ be vertices such that for each $i, r_{i}, s_{i} \in b d\left(F_{1}\right)$ or $r_{i}, s_{i} \in b d\left(F_{2}\right)$, and such that the parity condition is satisfied. Then the cut condition (54) is equivalent to the existence of paths (53).

This can be derived from Theorem 16(ii) as follows. First one argues that one may assume that, for some $t \leq k, r_{1}, r_{2}, \ldots, r_{t}, s_{1}, s_{2}, \ldots, s_{t}$ occur 
in this order clockwise around $\mathrm{bd}\left(F_{1}\right)$ and $r_{t+1}, r_{t+2}, \ldots, r_{k}, s_{t+1}, s_{t+2}, \ldots$, $s_{k}$ occur in this order clockwise around bd $\left(F_{2}\right)$.

We may assume that deleting $F_{1}$ and $F_{2}$ from the plane gives an annulus $S$. We extend $S$ by glueing the boundary of a cross-cap (a Móbius strip) at $\operatorname{bd}\left(F_{1}\right)$, and we augment $G$ by $t$ new edges over the cross-cap, connecting $r_{1}$ and $s_{1}, r_{2}$ and $s_{2}, \ldots, r_{t}$ and $s_{t}$, respectively. (This can be done in such a way that no two of them intersect.) Similarly, we glue the boundary of another cross-cap at bd $\left(F_{2}\right)$ and we put $k-t$ new edges over this cross-cap, connecting $r_{t+1}$ and $s_{t+1}, r_{t+2}$ and $s_{t+2}, \ldots, r_{k}$ and $s_{k}$, respectively. Let $G^{\prime}$ be the extended graph. So $G^{\prime}$ is an Eulerian graph embedded on a Klein bottle.

The cut condition now implies that the minimum size of a blocker in $G^{\prime}$ is equal to $k$. Hence by Theorem 16(ii), $G^{\prime}$ contains $k$ pairwise edgedisjoint orientation-reversing circuits. Since each of these circuits should contain at least one of the new edges over the cross-caps, and since there are $k$ such edges, in the original graph $G$ they give $k$ paths as required.

Another consequence is:

Corollary 16d. Let $G=(V, E)$ be an undirected planar graph, let $F_{1}$ and $F_{2}$ be two of its faces, and let $r_{1}, s_{1}, \ldots, r_{k}, s_{k}$ be vertices, such that $r_{1}, \ldots, r_{k}$ occur in clockwise order around bd $\left(F_{1}\right)$ and $s_{1}, \ldots, s_{k}$ occur in clockwise order around $b d\left(F_{2}\right)$, and such that the parity condition is satisfied. Then the cut conditions is equivalent to the existence of paths (53).

This can be derived by assuming that we obtain an annulus by deleting $F_{1}$ and $F_{2}$ (so we first make the unbounded face to a bounded face) and by glueing a cylinder (an annulus) so as to connect the boundaries of $F_{1}$ and $F_{2}$, in such a way that we obtain a Klein bottle. We extend $G$ over this cylinder by $k$ new edges connecting $r_{1}$ and $s_{1}, r_{2}$ and $s_{2}, \ldots, r_{k}$ and $s_{k}$, respectively. (This can be done in such a way that no two of them intersect.) This transforms $G$ to an Eulerian graph $G^{\prime}$ embedded on a Klein bottle.

The cut condition now implies that the minimum size of a blocker in $G^{\prime}$ is equal to $k$. Hence by Theorem 16(ii), $G^{\prime}$ contains $k$ pairwise edgedisjoint orientation-reversing circuits. Since each of these circuits should contain at least one of the new edges over the cylinder, and since there are $k$ such edges, in the original graph $G$ they give $k$ paths as required.

Despite analogies between Theorem 13 and Corollaries $16 \mathrm{c}$ and 16d, we do not see a common generalization. Karzanov [17], [18] obtained extensions to three or more holes. 
Acknowledgements. I thank an anonymous referee and Coelho de Pina for very helpful comments.

\section{References}

[1] A. Baudisch, Kommutationsgleichungen in semifreien Gruppen, Acta Mathematica Academiae Scientiarum Hungaricae 29 (1977), 235-249.

[2] R. Cole and A. Siegel, River routing every which way, but loose, in: Proceedings of the 25th Annual Symposium on Foundations of Computer Science, IEEE (1984), 65-73.

[3] G. Ding, A. Schrijver, and P.D. Seymour, Disjoint paths in a planar graph - a general theorem, SIAM Journal on Discrete Mathematics 5 (1992), 112-116.

[4] G. Ding, A. Schrijver, and P.D. Seymour, Disjoint cycles in directed graphs on the torus and the Klein bottle, Journal of Combinatorial Theory, Series B 58 (1993), 40-45.

[5] C. Droms, Isomorphisms of graph groups, Proceedings of the American Mathematical Society 100 (1987), 407-408.

[6] L.R. Ford and D.R. Fulkerson, Maximal flow through a network, Canadian Journal of Mathematics 8 (1956), 399-404.

[7] L.R. Ford and D.R. Fulkerson, A simple algorithm for finding maximal network flows and an application to the Hitchcock problem, Canadian Journal of Mathematics 9 (1957), 210-218.

[8] S. Fortune, J. Hopcroft, and J. Wyllie, The directed subgraph homeomorphism problem, Theoretical Computer Science 10 (1980), 111-121.

[9] A. Frank and A. Schrijver, Edge-disjoint circuits in graphs on the torus, Journal of Combinatorial Theory, Series B 55 (1992), 9-17.

[10] M. de Graaf and A. Schrijver, Grid minors of graphs on the torus, Journal of Combinatorial Theory, Series B, to appear.

[11] M. de Graaf and A. Schrijver, Decomposition of graphs on a surface, preprint, 1992.

[12] C. van Hoesel and A. Schrijver, Edge-disjoint homotopic paths in a planar graph with one hole, Journal of Combinatorial Theory, Series B 48 (1990), 77-91.

[13] T.C. Hu, Multicommodity network flows, Operations Research 11 (1963), 344-360.

[14] R.M. Karp, On the computational complexity of combinatorial problems, Networks 5 (1975), 45-68.

[15] A.V. Karzanov, Metrics and undirected cuts, Mathematical Programming 32 (1985), 183-198.

[16] A.V. Karzanov, Half-integral five-terminus flows, Discrete Applied Mathematics 18 (1987), 263-278. 
[17] A.V. Karzanov, Paths and metrics in a planar graph with three or more holes, I: Metrics, Journal of Combinatorial Theory, Series B, to appear.

[18] A.V. Karzanov, Paths and metrics in a planar graph with three or more holes, II: Paths, Journal of Combinatorial Theory, Series B, to appear.

[19] M. Kaufmann and K. Mehlhorn, On local routing of two terminal nets, Journal of Combinatorial Theory, Series B 55 (1992), 33-72.

[20] C.E. Leiserson and F.M. Maley, Algorithms for routing and testing. routability of planar VLSI-layouts, in: Proceedings of the 17th Annual ACM Symposium on the Theory of Computing, ACM (1985), 69-78.

[21] S. Lins, A minimax theorem on circuits in projective graphs, Journal of Combinatorial Theory, Series B 30 (1981), 253-262.

[22] M.V. Lomonosov, Combinatorial approaches to multiflow problems, Discrete Applied Mathematics 11 (1985), 1-94.

[23] J.F. Lynch, The equivalence of theorem proving and the interconnection problem, (ACM) SIGDA Newsletter 5 (1975), 3:31-36.

[24] K. Menger, Zur allgemeinen Kurventheorie, Fundamenta Mathematica 10 (1927), 96--115.

[25] H. Okamura, Multicommodity flows in graphs, Discrete Applied Mathematics 6 (1983), 55-62.

[26] H. Okamura and P.D. Seymour, Multicommodity flows in planar graphs, Journal of Combinatorial Theory, Series B 31 (1981), 75-81.

[27] R.Y. Pinter, River routing: methodology and analysis, in: Third CalTech Conference on Very Large Scale Integration, Springer-Verlag, Berlin, 1983, 141-163.

[28] B. Reed, N. Robertson, A. Schrijver, and P.D. Seymour, Finding disjoint trees in a planar graph in linear time, preprint, 1992.

[29] H. Ripphausen, D. Wagner, and K. Weihe, The vertex-disjoint Menger problem in planar graphs, preprint, 1992.

[30] N. Robertson and P.D. Seymour, Graph minors VI. Disjoint paths across a disc, Journal of Combinatorial Theory, Series B 41 (1986), $115-138$.

[31] N. Robertson and P.D. Seymour, Graph minors. VII. Disjoint paths on a surface, Journal of Combinatorial Theory, Series B 45 (1988), $212-254$.

[32] N. Robertson and P.D. Seymour, Graph minors XIII. The disjoint paths problem, preprint, 1986.

[33] A. Schrijver, Theory of Linear and Integer Programming, Wiley, Chichester, 1986.

[34] A. Schrijver, Homotopy and crossings of systems of curves on a surface, 
Linear Algebra and Its Applications 114/115 (1989), 157-167.

[35] A. Schrijver, Distances and cuts in planar graphs, Journal of Combinatorial Theory, Series B 46 (1989), 46-57.

[36] A. Schrijver, The Klein bottle and multicommodity flows, Combinatorica 9 (1989), 375-384.

[37] A. Schrijver, Decomposition of graphs on surfaces and a homotopic circulation theorem, Journal of Combinatorial Theory, Series B 51 (1991), 161-210.

[38] A. Schrijver, Disjoint circuits of prescribed homotopies in a graph on a compact surface, Journal of Combinatorial Theory, Series B 51 (1991), $127-159$.

[39] A. Schrijver, Disjoint homotopic paths and trees in a planar graph, Discrete \& Computational Geometry 6 (1991), 527-574.

[40] A. Schrijver, Edge-disjoint homotopic paths in straight-line planar graphs, SIAM Journal on Discrete Mathematics 4 (1991), 130-138.

[41] A. Schrijver, On the uniqueness of kernels, Journal of Combinatorial Theory, Series B 55 (1992), 146-160.

[42] A. Schrijver, Circuits in graphs embedded on the torus, Discrete Mathematics 106/107 (1992), 415-433.

[43] A. Schrijver, Graphs on the torus and geometry of numbers, Journal of Combinatorial Theory, Series B 58 (1993), 147-158.

[44] A. Schrijver, Classification of minimal graphs of given face-width on the torus, Report BS-R9203, CWI, Amsterdam (1992).

[45] A. Schrijver, Finding $k$ disjoint paths in a directed planar graph, SIAM Journal on Computing, to appear.

[46] A. Schrijver, Paths and circuits in directed graphs and combinatorial group theory, preprint, 1992.

[47] H. Servatius, Automorphisms of graph groups, Journal of Algebra 126 (1989), 34-60.

[48] P.D. Seymour, Four-terminus flows, Networks 10 (1980), 79-86.

[49] P.D. Seymour, Directed circuits on the torus, Combinatorica 11 (1991), 261-273.

[50] C. Wrathall, The word problem for free partially commutative groups, Journal of Symbolic Computation 6 (1988), 99-104.

CWI, Kruislaan 413

1098 SJ Amsterdam, The Netherlands and

Department of Mathematics

University of Amsterdam

Plantage Muidergracht 24

1018 TV Amsterdam, The Netherlands

Received January 21, 1993

Revised January 25, 1994 